Slavica

bruxellensia

\section{Slavica bruxellensia}

Revue polyphonique de littérature, culture et histoire

slaves

9 | 2013

Érotisme

\title{
Tolstoj et la question du sexe
}

\section{Michał Kruszelnicki}

Traducteur : Nicolas Litvine

\section{(2) OpenEdition}

Journals

Édition électronique

URL : http://journals.openedition.org/slavica/1254

DOI : 10.4000/slavica. 1254

ISSN : 2034-6395

Éditeur

Université libre de Bruxelles - ULB

Référence électronique

Michał Kruszelnicki, "Tolstoj et la question du sexe », Slavica bruxellensia [En ligne], 9| 2013, mis en ligne le 10 juillet 2013, consulté le 25 mai 2020. URL : http://journals.openedition.org/slavica/1254 ; DOI : https://doi.org/10.4000/slavica.1254

Ce document a été généré automatiquement le 25 mai 2020.

\section{(9) $\odot \Theta \Theta$}

Les contenus de Slavica bruxellensia sont mis à disposition selon les termes de la Licence Creative Commons Attribution - Pas d'Utilisation Commerciale - Pas de Modification 3.0 France. 


\title{
Tolstoj et la question du sexe
}

\author{
Michał Kruszelnicki \\ Traduction : Nicolas Litvine
}

Un homme, une femme, attirés l'un vers l'autre,
se lient par la luxure. (...) Leur amour signifie
qu'ils ne voient pas en l'autre leur être, mais leur
blessure, et le besoin d'être perdu : il n'est pas de
désir plus grand que celui du blessé pour une
autre blessure.
Georges Bataille, Le Coupable

\section{Introduction}

1 Le présent article vise à reconstruire la vision qu'avait Lev N. Tolstoj de l'amour physique et d'en faire une évaluation critique. J'ai choisi Anna Karenina (Anna Karénine, 1875-18771) pour point de départ de ma réflexion et comme l'une de ses sources principales, car c'est dans cette œuvre que Tolstoj consacre à la relation sexuelle une attention extraordinaire. Je m'attarderai cependant également sur des romans tels que Semejnoe sčastie (Le Bonheur conjugal, 1859), Vojna i Mir (Guerre et Paix, 1865-1869)et Krejtcerova sonata(Sonate à Kreutzer, 1889), ainsi que sur les essais philosophiques Tak čto že nam delă̌? (Ce qu'il faut faire, 1886) et Moja Vera (Ma Religion, 1884), afin de pouvoir montrer comment l'hostilité de Tolstoj envers les passions charnelles a évolué pendant les vingt ans séparant la publication de Guerre et Paix de celle de Sonate à Kreutzer.

Dans la première partie de mon étude, je replacerai Anna Karénine dans le cadre de l'histoire littéraire européenne $\mathrm{du} \mathrm{xIX}^{\mathrm{e}}$ siècle, et ce afin de montrer comment les questions clefs posées par ce roman sont issues de problématiques et de phénomènes ayant suscité un large intérêt à cette époque, tant en Europe occidentale qu'en Russie. Approfondissant la question de la crise religieuse vécue par Tolstoj vers le milieu de sa vie, je m'interrogerai sur l'effet que l'écriture d'Anna Karénine produisit sur lui, ainsi que sur le regard qu'il portait sur son œuvre la plus connue, eu égard au fait qu'il y évoque l'adultère, l'érotisme et la mort. J'attirerai également l'attention sur le rôle 
«d'éducateur de sa nation» qu'assuma Tolstoj, appelant à un retour aux valeurs religieuses de base à une époque de fascination grandissante pour les idées et modes de vie occidentaux. En vue d'asseoir mon argumentation, je me pencherai sur la vie sexuelle tourmentée et culpabilisante de Tolstoj et soutiendrai qu'elle doit être considérée comme un facteur important dans le développement de ses positions rigides.

3 Dans la seconde partie, j'analyserai certaines des stratégies stylistiques employées par Tolstoj dans Anna Karénine (il sera également fait référence à Guerre et paix, à Sonate à Kreutzer et aux principales œuvres philosophiques de Tolstoj), à l'appui de sa thèse voulant qu'un amour né de l'épanouissement physique et fondé uniquement sur celuici ne peut que devenir une force de destruction menant les amants au désespoir et à l'autodestruction.

4 Dans un troisième temps, je me pencherai sur les racines philosophiques et le caractère original des conceptions de Tolstoj quant à l'amour charnel. Je démontrerai que sa position en la matière fut influencée par les idées de certains philosophes, tant russes qu'européens et comparerai sa position avec l'érotisme sombre et compulsif professé par le Marquis de Sade puis développé par son " disciple », Georges Bataille, le célèbre penseur français, théoricien de la transgression, lequel a élaboré une philosophie complexe de l'érotisme de la transgression et de la mort pendant la Seconde Guerre mondiale. Je tenterai d'établir qu'Anna Karénine offre un regard remarquable sur le lien entre l'érotisme et la mort, entre le désir sexuel et la pulsion irrationnelle amenant l'être humain à mettre sa vie en danger, jusqu'au suicide.

5 Enfin, dans une conclusion exhaustive, je récapitulerai mes réflexions et en soulignerai les thèses clefs en vue d'inventorier les causes sociales, philosophiques et personnelles de l'aversion grandissante de Tolstoj pour l'amour charnel.

\section{Tolstoj, la Russie et les dangereuses idées du modernisme européen}

6 À l'époque où Tolstoj écrivait Anna Karénine, d'importantes questions sociales dominaient les pages des revues russes. Quelle devait être la position du pays à l'égard de l'esclavage? Comment devaient être éduqués les enfants et dans quelle mesure les écoles russes devaient-elle être influencées par les théories occidentales sur l'éducation? Dans quelle mesure la Russie avait-elle réellement besoin du "progrès » occidental qui se manifestait dans les domaines de la philosophie, de la politique ou encore de la technologie? Enfin, non des moindres, se posait la "question féminine " qui avait "principalement deux aspects : un aspect social et intellectuel et un aspect sexuel $»^{2}$. Les femmes devaient-elles avoir accès à l'éducation, au monde du travail et des affaires, ainsi qu'au service de l'État ? Étaient-elles amenées à avoir la même liberté que les hommes, de choisir leur conjoint, de faire une demande en mariage, voire de changer librement de partenaire sexuel?

7 L'atmosphère de la Décadence régnait sur la littérature européenne des dernières décennies $\mathrm{du} \mathrm{XIX}^{\mathrm{e}}$ siècle, créant un milieu favorable au développement de l'image de femmes libérées, sexuellement actives, voire débauchées, tant dans le domaine artistique que dans la culture populaire. En fait, beaucoup des déviants dépeints dans la fiction décadente étaient des femmes. Pour illustrer ce propos, il suffira de mentionner 
quelques œuvres liées à ce mouvement : dans La Faenza (1883) de Jean Moréas, une mère séduit son propre fils; dans Monsieur Vénus (1884)de Rachilde, une femme se plaît à battre sa "maitresse " masculine; dans Méphistophéla (1893) de Catulle Mendès, un sabbat lesbien atteint son point culminant avec la castration d'hommes; dans Le Jardin des supplices (1899) d'Octave Mirabeau nous rencontrons Clara, prédatrice et corrompue, qui prend plaisir à faire visiter à un jeune homme le jardin éponyme où l'on peut faire l'expérience de toute sorte de tortures élaborées; etc. D'autres figures d'amantes dangereuses furent proposées au lecteur par Edgar Allan Poe (Bérenice, 1835; Ligea, 1838), Oscar Wilde (Salomé, 1891), Algernon Swinburne (Anactoria, 1866) et bien d'autres ${ }^{3}$.

8 La culture moderniste de l'Europe fut aussi significativement influencée par la toute jeune littérature scandinave. Des auteurs tels qu'August Strindberg, Henrik Ibsen et Knut Hamsun firent des relations érotiques entre hommes et femmes l'un des principaux thèmes de leurs travaux. Tandis qu'Ibsen est généralement perçu comme un précurseur des tendances libérales et féministes européennes, Strindberg, dans son très controversé roman autobiographique Le Plaidoyer d'un fou $(1893)^{4}$, qualifiait les femmes émancipées de «folles » et de " demi-femmes » et, déchiré entre adoration et haine du sexe opposé, se plaignait amèrement de l'impuissance des hommes face au pouvoir de séduction des femmes. Il peut être soutenu que les auteurs décadents avaient une relation aux femmes ambivalente : d'une part ils étaient attirés par leur sexualité tandis que, d'autre part, elle leur répugnait. Les ouvrages précités circulaient largement en Europe et étaient évoqués dans les salons littéraires. Ils apportaient un changement à la vision des femmes et contraignaient ainsi les intellectuels européens à réévaluer la nature des relations sociales et maritales.

Considéré comme controversé, voire révolutionnaire, en Occident, ce type de littérature paraissait bien plus choquant encore en Russie. Déjà avant que la fiction décadente n'atteigne son point culminant, Tolstoj s'était montré fort suspicieux à l'égard des idées nouvelles venues de l'Ouest. On trouve de nombreux indices de l'influence de romans français antérieurs dans l'œuvre de Tolstoj, particulièrement en ce qui concerne le thème de l'adultère. Durant son séjour à Paris en 1857, il allait souvent à l'opéra et au théâtre, il lisait les classiques de l'époque (Gustave Flaubert, Stendhal, etc.) et fut particulièrement "dégoûté " par la "dépravation » d'Honoré de Balzac. L'une de ses sources d'inspiration les plus importantes s'avérera toutefois le roman que Flaubert venait de publier, Madame Bovary (1857), qui présente un personnage de femme frustrée, habitée par des fascinations artistiques morbides et qui ne peut cultiver sa passion érotique en raison d'une étiquette sociale restrictive. ${ }^{5} \mathrm{~A}$ ces "nouveautés» occidentales Tolstoj réagissait en affichant ses opinions ultraconservatrices sur l'éducation et le mariage. En matière de droits des femmes, Tolstoj était d'avis que le rôle d'une femme était d'être une épouse fidèle et une mère fertile. Il affichait une attitude ouvertement hostile envers les femmes «éduquées » et les raillait avec malveillance dans son journal : «Je vois des étudiantes avec leurs livres et cahiers, courant d'une leçon à l'autre. Des femmes peintres, des musiciennes. Elles savent tout faire. Et, telles des singes, elles ont imité les hommes en tout. ${ }^{6}$ Comme nous aurons l'occasion de le constater par la suite, Tolstoj considérait que, pour une femme, la vie ne devait commencer qu'après qu'elle ait mis au monde les enfants de son mari. 
10 Tolstoj, bien plus que Fëdor M. Dostoevskij, se sentait responsable du «salut » de la "Sainte Russie » et de la défense de sa nation contre les aberrations trompeuses en provenance de l'Europe. Il est toutefois intéressant de noter que ni Tolstoj ni Dostoevskij n'ont vécu conformément aux valeurs qui étaient les leurs. Un grand nombre de biographies ont mis en évidence les nombreuses faiblesses de Tolstoj et ont montré à quel point il s'était écarté des valeurs qu'il prêchait. ${ }^{7}$ Une des principales autocritiques apparaissant de son journal était son incapacité à résister à l'attrait sexuel des femmes. Tout au long de sa vie, les rapports sexuels choquèrent Tolstoj, le fascinant et le repoussant tout à la fois. Même après le changement intérieur qui suivit la publication d'Anna Karénine, lorsqu'il s'efforça de mener une vie qui reflétait réellement ses idées, il ne le fit que parce qu'il ressentait le besoin d'un rituel de purification chaque fois qu'il se préparait à s'engager dans une nouvelle relation amoureuse. Tolstoj bâtit avec sa femme, Sofija Andreevna Bers, une relation passionnée mais douloureuse et marquée du sceau de la méfiance et du ressentiment mutuel. Ces relations torturées au sexe opposé et ces différents conjugaux ont façonné les vues de Tolstoj quant à l'amour et au mariage.

11 La crise existentielle et le changement spirituel finirent par amener Tolstoj à la conclusion que la vertu authentique était faite de travail simple, d'une vie faite de sacrifice de soi, de foi aveugle en Dieu et, bien sûr, d'abstinence sexuelle. Cette vision ascétique du monde se fit jour pour la première foi dans la Ispoved'(Ma Confession, 1882) de Tolstoj (écrite deux ans après Anna Karénine), à propos de laquelle Tim Cook déclare à raison qu'elle « signalait le début de la transformation de Tolstoj, l'écrivain admire en Tolstoj le sage révéré, révélant son intérêt croissant pour les questions spirituelles et religieuses ${ }^{8}$. Ma Confession est l'autoportrait dramatique d'un artiste qui, ayant vécu toute sa vie parmi les écrivains «éduqués » et les intellectuels verbeux, commence à comprendre combien le fait "d'écrire des livres " et "d'éduquer les autres » était dépourvu de sens, absurde et faux aussi longtemps que l'on n'avait pas la réponse à « la question de la vie la plus simple » : comment vivre? "qu'est-ce qui est bon et qu'est-ce qui est mauvais? ${ }^{9} \mathrm{Ma}$ Confession reflétait le mécontentement croissant de Tolstoj par rapport à son existence jusqu'alors «superficielle " - ainsi qu'il se l'assénerait de manière répétée - et anticipait sur sa recherche ultérieure d'un nouveau sens de la vie dans le respect des enseignements du Christ et la sagesse simple du peuple russe.

Bien que les années aient passé, Tolstoj ne trouva pas ce qu'il cherchait. Sa vie se termina en dépression. Déjà plusieurs années avant sa mort, Tolstoj écrivait dans son journal que sa vie était entièrement dépourvue de sens. Tous ses efforts pour mener une vie chrétienne échouèrent complètement, peut-être - ainsi que le suggéra Fëdor Stepun - parce qu'il « lui manquait l'authentique expérience chrétienne, parce que la vérité qu'il défendait n'était pas celle du Christ, mais la sienne propre ${ }^{10}$.

Après Ma Confession, Tolstoj renonça à assumer le rôle de premier écrivain de son peuple et s'investit dans le domaine de la pensée religieuse, souhaitant proclamer sa propre vision du christianisme. Cette vision s'avéra très éloignée des enseignements traditionnels de l'Église orthodoxe. À partir des années 1880, Tolstoj écrivit un énorme corpus d'articles et d'essais (dont Kritika dogmatičeskogo bogoslovija[Critique de la théologie dogmatique, 1880] et Četveroevangelie : Soedinenie i perevod četypëkh Evangelij[Les quatre Évangiles réunis et traduits, 1881]) fort critiques au sujet de l'Église orthodoxe qui, disaitil, s'était écartée de l'idée originelle du christianisme. En représailles, le Saint Synode l'excommunia en $1901 .^{11}$ En 1884, Tolstoj publia un traité religieux, Moja Vera (Ma 
religion), dans lequel il tentait d'établir des principes chrétiens pour guider sa foi et sa vie - pour le voir bientôt interdit en Russie. Au milieu des années 1880, Tolstoj bénéficiait d'un statut de sage quasi-divin et sa maison de Jasnaja Poljana devint un temple visité par toute sorte de personnes à la recherche de conseil et de guidance morale.

14 Si l'on devait se représenter le travail de Tolstoj sur Anna Karénine, le principal thème du roman étant la trahison conjugale et la passion charnelle, on ne pourrait penser ce processus que comme une lutte épuisante rendant le travail particulièrement pénible à poursuivre dans la mesure où il impliquait de nier les valeurs qui étaient celles de Tolstoj. Pour l'âme religieuse de Tolstoj, la passion sexuelle et le plaisir du corps prenant le dessus sur l'amour spirituel et le respect de la tradition incarnée par le mariage étaient impossibles à accepter. Anna Karénine est donc condamnée à un destin tragique, à l'infamie et à la chute finale.

\section{La vision sombre de l'amour charnel dans Anna Karénine}

Lors de la première rencontre d'Anna et de Vronskij, le lecteur assiste à la naissance de leur passion. Cette passion éclot toutefois sous le signe de la mort. Dans un accident macabre, un employé de gare perd la vie sous les roues du train. Cette mort est significative à au moins trois égards. Dans l'imaginaire artistique de Tolstoj, le train est un symbole du "progrès » moderne lequel perturbe le mode de vie traditionnel, russe. En tant qu'invention étrangère, le train peut également être associé aux idées dangereuses faisant irruption en Russie depuis l'Ouest, telles que la libération de la femme. Enfin, le train peut renvoyer à la mort en tant que celle-ci est liée à la passion illicite. ${ }^{12}$ La scène de mort violente en début de roman préfigure le sort de l'amour d'Anna et de Vronskij et suggère pour la première fois l'existence d'un lien fort entre érotisme et mort, entre sexualité et violence.

16 Peu après, nous retrouvons Anna au bal donné dans la propriété des Ščerbatskij. Elle n'est pas habillée en lila - comme l'avait supposé Kitty - mais porte une robe noire. Alors que les couleurs envisagées par Kitty représentent la pureté et l'innocence, le noir évoque les forces maléfiques, la tentation infernale et le péché. Et, en effet, lorsque Kitty observe Anna dansant avec Vronskij, elle perçoit «une séduction étrange, presque infernale ${ }^{13}$. Cette chose " séduisante » chez Anna est son pouvoir sexuel sur les hommes, pouvoir dont elle est immédiatement consciente. « Pour Kitty (...) Anna est une déesse, mais une déesse païenne, voire chtonienne. Kitty, depuis son cocon familial et enveloppée par sa propre vertu, éprouve pour elle de l'attraction mais aussi, au fond, de la répulsion. $»^{14}$ Les termes « étrange » et «infernale » indiquent assez nettement qu'un esprit dangereux et mauvais s'est éveillé en Anna.

17 La chute fatale d'Anna dans la passion se produit sur le chemin de retour depuis Moscou vers Saint-Pétersbourg. Les cahots du train, la tempête déchainée derrière les fenêtres et les soudaines bouffées de chaleur d'Anna sont autant de métaphores de son état intérieur. Tentant de rationaliser son comportement, elle commence à jouer avec un coupe-papier. Elle « le serre [fermement] dans ses mains » [AK, I, 104] et, finalement, presse la surface froide de sa lame sur sa joue chaude. Appréhendée d'un point de vue symbolique, cette scène attire à nouveau l'attention sur la nature de sa passion. Le couteau est une arme mortelle, il représente une menace, apporte la destruction, il 
peut aussi représenter la sexualité libérée des femmes. L'image d'une femme jouant avec un couteau peut fort bien représenter la soumission des hommes au pouvoir qu'elle a sur eux, le pouvoir de faire d'eux les victimes de son désir. Plus loin nous voyons Anna dans son lit, écoutant les ronflements de son mari. Elle est pourtant loin d'être somnolente. En fait, elle fixe le plafond, de ses «les yeux ouverts et croyant les sentir briller dans l'obscurité » [AK, I, 152]. Un étrange sourire sur le visage d'Anna le fait paraître comme hanté par des forces maléfiques.

Ce qui suit est la scène - fort admirée de la critique littéraire - d'amour/séduction au cours de laquelle Tolstoj compare l'acte sexuel à un meurtre, l'homme à un boucher et la femme à sa victime. Vronskij se dresse devant Anna, "pâle et tremblant » [AK, I, 153], tentant en vain de la réconforter tandis qu'Anna est assise sur un canapé, la tête baissée, incapable de supporter son sentiment de culpabilité et d'humiliation. Vronskij tente désespérément de la convaincre du "bonheur " qui leur est venu, mais Anna l'interrompt, dédaigneuse et horrifiée, elle murmure : « au nom du ciel, pas un mot, pas un mot de plus» [AK, I, 154].

19 Aux yeux de Tolstoj, rien ne peut justifier l'acte d'amour d'Anna et de Vronskij. La scène crée une atmosphère déchirante de désastre moral et de fatalité quasiment physique pesant sur les amants. Anna et Vronskij sont condamnés à l'instant même où ils brisent l'équilibre naturel de la famille de la première. Tolstoj ne savait que trop bien le cauchemar que devait être, pour une femme comme Anna, la vie avec un automate humain tel que son mari - Aleksej Aleksandrovič. Mais le tromper revient, chez Tolstoj, à " enfreindre les règles », ce qui implique que "vient un châtiment, qui peut être plus ou moins cruel $»^{15}$. Aux yeux de Tolstoj, le sacrement du mariage a une valeur indiscutablement sacrée. En dépit de ses propres déboires conjugaux, Tolstoj maintenait une position rigide quant à l'indissolubilité du mariage, rejetant l'idée de divorce. Pour Levin, alter ego de Tolstoj dans Anna Karénine, le mariage et la vie de famille ne sont pas seulement les choses les plus importantes, mais aussi les seules valeurs le retenant de mettre fin à ses jours. Ainsi que Donna Orwin a pu le soutenir :

Le mariage est vu dans cette œuvre comme apportant un élément de chasteté ou de régulation à la sauvagerie de la passion sexuelle. (...) La passion d'Anna et de Vronskij, puissante et authentique, mène au désastre, tandis que l'amour également « aveugle » de Kitty et de Levin est légitime car il sert la cause de la perpétuation de l'espèce humaine. ${ }^{16}$

Selon Tolstoj, une authentique relation maritale ne devrait pas être fondée sur la satisfaction physique (ce n'est pas sans raison que Levin se refuse même à «utiliser le mot prelest ["charme" - note du traducteur] pour décrire son mariage avec Kitty, alors qu'il l'emploie à l'égard d'Anna durant sa visite $\aleph^{17}$ ), mais sur quelque chose d'entièrement opposé : concevoir des enfants et leur assurer une bonne éducation. Les prémonitions éthiques figurant dans les œuvres de Tolstoj des années 1860 et 1870 sont développées sur le plan philosophique dans Ma religion, Ce qu'il faut faire et Sonate à Kreutzer. Dans Ce qu'il faut faire, Tolstoj décrit son idéal éthique comme une tâche impressionnante "d'oubli de soi dans les douleurs de l'enfantement et de sacrifice de soi dans l'éducation de l'enfant, qui n'est accessible qu'aux femmes ${ }^{18}$.

21 Anna Karénine ne trouve toutefois pas la satisfaction dans la maternité, ce qui est rendu manifeste par ce qu'Edwina Cruise a justement évoqué comme une attitude « erratique » à l'égard de son fils Serëža, adoptée par Anna dès avant sa liaison avec Vronskij. ${ }^{19}$ Anna rejette la perspective d'une nouvelle grossesse, pensant que cela ferait obstacle aux plaisirs qu'elle partage avec Vronskij. Aux yeux de Tolstoj, cela en fait 
automatiquement une "femme déchue». Lorsque dans Sonate à Kreutzer, l'épouse après avoir donné naissance à des enfants - commence à prendre des mesures de contraception sur le conseil de son médecin, son mari irrité - Pozdnyšev - condamne ce choix car - de son point de vue - il peut libérer sexuellement sa femme. Et, tout comme Tolstoj, il juge que cela n'est pas bon. Dans Ce qu'il faut faire, le mépris de Tolstoj pour les femmes «qui s'occupent uniquement de taille, de tournure, de coiffure, de coquetterie amoureuse $»^{20}$ atteint son sommet, concomitamment avec son exaltation des femmes qui se conforment avec empressement à « la loi éternelle » et à « la volonté de Dieu » en assumant des grossesses (de préférence) nombreuses. Si l'on résiste à cet idéal et que le sacrifice de soi dégénère en un penchant pour l'épanouissement de soi, comme dans le cas d'Anna ou de Pozdnyšev, la catastrophe devient imminente.

Succombant au désir sexuel, Anna change profondément. Plus sa passion pour Vronskij devient forte, plus elle méprise son mari Aleksej Aleksandrovič. Elle commence d'abord à remarquer ses défaut, ses grandes oreilles, puis elle s'agace de sa voix « odieuse (...) dont elle connaissait toutes les intonations » [AK, I, 213]. Elle finit par haïr tout ce qu'il représente : l'hypocrisie, la froideur et le désintérêt pour ses sentiments, l'étroitesse d'esprit, l'idiotie, la pédanterie comique et l'égoïsme. À l'approche de la fin dramatique du roman, Anna vit encore plus de changements. Elle devient jalouse à l'égard de Vronskij, provoque des querelles et le harcèle d'accusations irrationnelles. Son visage porte de plus en plus la marque de la malice. À la grande horreur de Dolly, qui vient lui rendre visite, elle ne s'inquiète guère de ses enfants et prend de la morphine ainsi que de l'opium mêlé à son vin afin d'apaiser sa conscience. Elle est la première héroïne de Tolstoj à fumer des cigarettes. Seule son adoration pour Vronskij rend sa vie digne d'être vécue. Ne se sentant pas assez attirante pour lui, elle flirte avec Levin pour tirer vengeance des infidélités dont elle accuse Vronskij. Reginald Christian suggère - à raison - qu'Anna « devient un parasite, exploitant son amant » et que "son appétit excède sa capacité à contrôler celui-ci $»^{21}$.

Bien que la puissance destructrice de la passion charnelle soit illustrée par l'examen de la vie intérieure agitée d'Anna, les sentiments de Vronskij ne demeurent pas inchangés depuis la naissance de son amour pour Anna. Il est un sentiment qu'il tente constamment de refouler et de nier. Tolstoj le caractérise ainsi: «une étrange sensation de dégoût et de répulsion qu'il ne pouvait définir. Pour qui l'éprouvait-il ? ... Pour Aleksej Aleksandrovič, pour lui-même, pour le monde entier? $»^{22}$. De mon point de vue, ce qu'il ressent à propos de son adultère avec Anna ne peut être simplement considéré comme de la culpabilité. Cela doit plutôt être considéré comme un rejet de la sexualité qui réduit hommes et femmes en esclavage. Le protagoniste de Sonate à Kreutzer, se rappelant les réconciliations charnelles qui suivaient ses violentes querelles avec sa femme, exprime également son écœurement absolu envers cette fonction «basse » de la sexualité. Il dit : « en théorie cet amour est idéal, éthéré, en pratique c'est quelque chose de misérable et de malpropre dont on ne peut parler sans dégoût et sans honte $»^{23}$.

Dans Anna Karénine, Tolstoj avance nombre d'autres arguments à l'encontre des tentations de l'attirance sexuelle. À l'opéra, Vronskij regarde Anna et la trouve plus belle que jamais auparavant, mais désormais « cette beauté et cette élégance étaient précisément ce qui l'irritait » [AK, II, 173]. Tolstoj semble affirmer que, la fascination charnelle étant le fondement même de l'amour d'Anna et de Vronskij, il sera tôt ou tard empoisonné par la jalousie et la méfiance réciproque. On notera que le même thème est 
à nouveau présent dans Sonate à Kreutzer ${ }^{24}$, où le personnage principal, après avoir mené la vie d'un séducteur et d'un "voluptueux ", épouse une femme, mais il ne le fait que parce qu'il la désire comme un simple objet sexuel. C'est pourquoi il est constamment torturé par la jalousie et la suspicion qui le mènent finalement à poignarder sa femme à mort.

Dans Anna Karénine, Tolstoj montre comment le désir sexuel pervertit l'imagination et mène à la catastrophe de la jalousie, de l'hypocrisie, de la luxure refoulée et de la honte grandissante. À l'inverse, l'amour privé de sexualité amène à un épanouissement paisible. Dans Sonate à Kreutzer, Tolstoj écrit : «C'eût été un amour platonique, puisque c'est de celui-là que nous parlons, et non sensuel, il aurait dû, cet amour platonique, se traduite en paroles, en entretiens » [SK, 73]. Tolstoj attendait de l'amour entre les sexes qu'il s'identifie à l'amitié, le sexe n'étant qu'un moyen de reproduction. Unis exclusivement par la fascination physique, les partenaires ne peuvent que suivre le chemin d'une déception et d'une distance grandissante, comme c'est le cas de ceux des personnages de Tolstoj qui se sont écartés du chemin de la décence et de la chasteté conjugale : Pierre et Hélène (Kuragin) Bezukhov dans Guerre et Paix, Anna et Vronskij dans Anna Karénine, et Pozdnyšev et sa femme dans Sonate à Kreutzer.

Tolstoj ne cesse de répéter qu'après que le désir sexuel a été satisfait, l'objet qui l'a suscité apparaît soudain comme banal, ordinaire, voire répugnant. Dans sesCarnets, Tolstoj analyse encore et encore cette impression étrange et, dans Anna Karénine, il le présente comme un mécanisme dont le mouvement inéluctable finit par faire obstacle à la relation d'Anna et de Vronskij. Déjà après la scène de la séduction, Vronskij est décrit comme "pas entièrement heureux ». C'est parce qu'après la relation sexuelle Anna, jusqu'alors divinisée, "apparaît entièrement terrestre et, dès lors, incapable de répondre aux attentes de Vronskij ${ }^{25}$.

Il n'est donc pas difficile de comprendre pourquoi, dans Anna Karénine et Sonate à Kreutzer, Tolstoj compare les rapports sexuels à un crime. L'acte sexuel prive une personne de choses qui ont, pour Tolstoj, une grande valeur : l'innocence, la spiritualité et la spontanéité. Tombée en disgrâce, Anna ne peut que voir Vronskij s'écarter d'elle en conséquence. Rendue folle par une « besoin vague de vengeance » [AK, II, 317] elle se dirige progressivement vers une mort par suicide. Sa dernière réflexion est :

Peut-il encore s'établir entre lui et moi des relations qui me donnent, je ne dis pas

$\mathrm{du}$ bonheur, mais des sensations qui ne soient pas une torture? Non, se réponditelle sans hésiter, la scission entre nous est trop profonde ; je fais son malheur, il fait le mien, nous n'y changerons plus rien! ${ }^{26}$

La relation d'Anna et de Vronskij est alors visuellement comparée à «la vis qui s'est dévissée $\aleph^{27}$, à quelque chose de surexploité, d'épuisé, sans perspective de développement ultérieur. Anna n'a donc pas d'autre issue. Le train mutilant son corps symbolise la mort qui sanctionne le péché d'érotisme égaré incarné par la passion d'Anna et de Vronskij. Effondré moralement, Vronskij sombre dans le désespoir et se porte volontaire pour aller combattre les Turcs. Il cherche ainsi l'oubli et, probablement, la mort. 


\section{Anna Karénine, la littérature et la philosophie. Les sources de la pensée de Tolstoj}

La vision de l'amour soutenue par Dostoevskij et - vers la fin de son œuvre - par Tolstoj se forgèrent dans le cadre de polémiques avec celle défendue par Vladimir Solov'ëv, un des plus grands philosophes, poètes et romanciers russes. Alors que Tolstoj, dans Sonate à Kreutzer, condamnait totalement l'amour charnel, Solov'ëv, dans Le Sens de l'Amour (1894), attribuait au sexe un rôle significatif dans le processus d'unification des partenaires dont la fusion dans un acte physique permet à la relation d'évoluer vers une authentique communion spirituelle. Contrairement à Tolstoj, Solov'ëv ne concevait pas la reproduction comme unique sens de l'amour: "L'amour charnel et la propagation des espèces se trouvent en proportion inverse l'un de l'autre: le plus unissant est le premier, la plus faible est la seconde $»^{28}$. L'amour - bien que construit sur la base de l'attirance charnelle - concerne plus le développement spirituel par lequel l'individu conçoit en l'autre une image de Dieu, devenant ainsi un avec son partenaire. Selon Solov'ëv, l'amour exige de mieux considérer l'autre que soi-même. Il exige le sacrifice de son égoïsme au nom de «l'autre»: «[L'amour] implique la personnalité individuelle, en vertu de laquelle précisément cette personne de l'autre sexe possède une signification absolue pour le partenaire, comme unique et irremplaçable, comme une fin en soi. ${ }^{29}$ De ce point de vue, l'amour devient l'unique puissance à même de transcender l'égoïsme inhérent à l'être humain, il est capable de vaincre l'égoïsme et ainsi de racheter une réalité déchue.

Tolstoj rejoignait cette opinion et s'en servait comme argument dans sa quête de purification des relations conjugales humaines. La relation de Kitty et de Levin dans Anna Karénine est censée servir d'exemple d'un amour idéal, tandis que l'amour charnel d'Anna et de Vronskij était condamné comme succombant aux tentations trompeuses du Malin. L'amour d'Anna est ainsi représenté comme basé exclusivement sur l'égoïsme et comme aveugle à la valeur spirituelle, divine, de l'autre. Il l'isole de Vronskij au lieu de l'en rapprocher. Dans Ma Religion, Tolstoj déclarera :

Il faut écarter tout ce qui excite à la volupté, éviter de donner l'éveil à la volupté, et, s'étant uni avec une femme, ne jamais l'abandonner sous aucun prétexte; car cet abandon produit la débauche. Les femmes abandonnées séduisent d'autres hommes et introduisent la débauche dans le monde. La sagesse de ce commandement me frappa. Il supprime tout le mal qui, dans le monde, est la conséquence des rapports sexuels. Les hommes, convaincus que la licence des rapports sexuels aboutit aux querelles, éviteront tout ce qui donne éveil à la volupté, et, sachant que la loi humaine est de vivre par couples, - s'uniront en couples sans jamais enfreindre cette union. Tout le mal provenant des dissensions que l'attrait sexuel occasionne sera supprimé, parce qu'il n'y aura plus ni hommes ni femmes privés de rapports sexuels. ${ }^{30}$

31 Dans Sonate à Kreutzer, Tolstoj exprime sa conviction que les passions font obstacle à la réalisation $\mathrm{du}$ «but de l'homme comme de l'humanité entière, (...) bonheur » qui doit être atteint par «l'union des êtres qui composent l'humanité », mais aussi que «la dernière, la plus forte, la pire, [est] l'amour sensuel » [SK, 80-81] Mon intention est de soutenir que c'est dans ce roman que l'influence de la philosophie d'Arthur Schopenhauer sur Tolstoj est la plus manifeste. Après avoir terminé Guerre et Paix, Tolstoj avait étudié la pensée de Schopenhauer par le biais de son ouvrage Die Welt als Wille und Vorstellung (Le Monde comme volonté et comme représentation, 1819) dans lequel 
Schopenhauer identifie la "volonté » humaine au mal qui conduit au malheur et à l'insatisfaction éternelles. La "volonté » est mauvaise parce que l'on ne peut y mettre fin, parce qu'elle reste toujours inassouvie. Par conséquent, Schopenhauer estimait que l'homme ne pouvait être sauvé de son élan insatisfait qu'en refrénant sa volonté et en opposant un refus à toutes ses ambitions, ses passions et ses instincts. Dans le même temps, le philosophe n'était que trop conscient que l'ascèse totale était impossible - la volonté étant la vie elle-même - et il voyait donc le Monde et l'existence humaine comme un cauchemar perpétuel que l'on ferait mieux de quitter en se réveillant. Ceci amenait Schopenhauer à faire l'éloge du suicide comme une voie acceptable d'échapper aux tourments de l'existence. On peut soutenir que cette vision pessimiste du destin humain a influencé Tolstoj dans Anna Karénine et Sonate à Kreutzer. L'influence de Schopenhauer sur Tolstoj est particulièrement sensible dans sa condamnation de l'amour charnel comme l'une des manifestations les plus fortes des maux affectant la volonté humaine.

32 Je suis également convaincu que la philosophie sur laquelle est fondé Anna Karénine correspond à certains égards à la philosophie moderne de l'érotisme, particulièrement celle développée par Bataille. Ce penseur français très influent était fasciné par les moments extrêmes et transgressifs de l'existence humaine. Bataille soutenait que les expériences d'horreur extrême, de rire incontrôlable, d'ivresse, de luxure et de risque rapprochaient l'être humain du sacrum - l'indicible Absolu qui nous met dans un état de continuité divine par rapport à notre existence momentanément inscrite dans la globalité de l'Être. Chez Bataille, l'érotisme et l'acte sexuel se conçoivent comme les meilleurs exemples de ce dangereux oubli de soi et du désir inconscient de l'homme d'y accéder. Dans un passage évocateur du Coupable, Bataille compare l'acte sexuel à une expérience mystique. De mon point de vue, cette vision complète adéquatement les opinions de Tolstoj sur le sexe et l'érotisme: "L'expérience mystique diffère de l'érotique en ce qu'elle réussit pleinement. L'excès érotique aboutit à la dépression, à l'écœurement, à l'impossibilité de persévérer, et le désir inassouvi parfait la souffrance. L'érotisme excède les forces humaines. $\|^{31}$ La pensée de Bataille peut s'avérer pertinente dans l'étude de la philosophie du sexe de Tolstoj car elle fournit un éclairage sur le lien entre l'érotisme et la mort tel que représenté dans Anna Karénine. Bataille partageait la conviction du Marquis de Sade que le désir sexuel incontrôlé était inséparable de la violence, du désir profond de blesser son partenaire et d'être blessé. Bataille écrit :

Un homme, une femme, attirés l'un vers l'autre, se lient par la luxure. La communication qui les mêle tient à la nudité de leurs déchirures. Leur amour signifie qu'ils ne voient pas en l'autre leur être, mais leur blessure, et le besoin d'être perdu : il n'est pas de désir plus grand que celui du blessé pour une autre blessure. ${ }^{32}$

Bataille, qui était attentif à distinguer la fonction reproductive humaine et l'érotisme, voyait ce dernier comme n'ayant aucun «but» si ce n'est la mort: en ayant des relations sexuelles, l'individu accède à un aperçu de l'instant de sa mort (oubli de soi, sensation ambivalente de continuité divine et d'immédiateté de l'existence) et c'est ce qui fait l'attrait de l'érotique. À long terme, cependant, la frénésie sexuelle conduit à l'incomplétude et à la frustration parce que le partenaire sexuel ne peut jamais entièrement combler nos besoins, le but ultime de notre désir érotique étant la fusion permanente avec le partenaire, d'entrer irrévocablement dans la continuité sacrée et intemporelle... et de cesser d'exister. Cette unicité avec l'autre ne peut être atteinte 
qu'au prix de la violation de l'autonomie et de la destruction de l'intégrité physique de soi et de son/sa partenaire. ${ }^{33}$

Je me risquerais à formuler l'hypothèse que lors de l'écriture d'Anna Karénine et de Sonate à Kreutzer, Tolstoj n'était guère éloigné de la pensée de Bataille. Les exigences érotiques d'Anna sont d'autant plus destructrices que - comme le dit justement Edward Wasiolek - « elles ne peuvent êtres satisfaites " ${ }^{34}$. On peut dès lors se demander s'il n'y a pas dans l'amour d'Anna, bien avant son suicide, quelque chose qui recherche la mort. N'y a-t-il pas un élément de sadomasochisme dans sa passion? Le célèbre passage relatant le retour d'Anna de Moscou à Pétersbourg fait apparaitre une terrible tempête à l'extérieur de son wagon. Et pourtant Tolstoj écrit que: «jamais l'horreur de la tempête n'avait paru si belle à Anna » [AK, I, 107]. Peut-être cette tempête exprime-telle le désir d'Anna de tomber dans l'oubli de soi ou même.... dans la mort. Il semblerait alors que, d'une part, Tolstoj tente dans Anna Karénine de rejeter le sexe en tant qu'instrument de mort mais que, d'autre part, il est manifestement conscient de l'aspect transgressif et dangereux de l'érotisme, ainsi qu'on le voit dans la manière dont il associe l'idée d'accomplissement érotique avec celle de transgression de la norme sociale (adultère, refus de la maternité) et avec la perspective de "l'ego » rationnel capable de vivre l'authenticité, la continuité et l'intensité de l'existence seulement après avoir effectué un saut dans l'abîme, et ce au risque de son propre avilissement moral, de sa dégradation psychologique, ou même de sa mort.

Mais, plutôt que d'explorer l'abîme avec la fascination de Bataille ou des décadents, Tolstoj s'arrête et rejette l'érotisme dans son ensemble. Pour une âme religieuse, orthodoxe, comme celle de Tolstoj, la vie doit toujours triompher de la mort. Il peut donc être soutenu que Tolstoj ne condamnait pas Anna pour son adultère, mais pour avoir choisi la mort (l'érotisme) plutôt que de tenter de construire une vie dans le mariage et l'éducation des enfants (la chasteté). À moins que le sexe ne mène les partenaires à une évolution spirituelle, à la reproduction et à la consolidation de la famille - affirme Tolstoj - il reste une manifestation d'égoïsme diabolique et une menace mortelle pour la «bonté » inhérente à l'être humain. Chaque fois que l'on lit Tolstoj à propos de la "bonté » intérieure ou du « caractère naturel » de l'homme, on constate la fascination constante de l'écrivain pour l'œuvre de Jean-Jacques Rousseau. ${ }^{35}$ $C^{\prime}$ 'est un peu comme Rousseau que le Tolstoj tardif en vient à envisager le sexe comme une "invention» traitresse de la culture moderne qui pervertit le déroulement «naturel» de la vie. Ce dualisme civilisation/nature lié au dualisme amour charnel/ platonique apparaît clairement dans Anna Karénine. Barbara Lönnquist remarque que «l'histoire d'Anna et de Vronskij se développe dans une symbolique du fer, de la machine, et de la création artificielle ", alors que la relation érotique de Levin avec Kitty « est comme une révélation graduelle de la vie, une vie faite de tradition et de l'expérience de ses prédécesseurs. Son amour pour sa femme et son fils s'inscrit dès lors dans des schémas qu'il n'a pas créé et qui sont dès lors éprouvés ${ }^{36}$.

À l'inverse de Levin, Anna prend le parti de la civilisation moderne/de la mort lorsqu'elle s'engage dans un amour charnel et refuse d'avoir plus d'enfants, que ce soit avec son mari ou avec Vronskij. Ce faisant, elle « se dresse contre la vie naturelle avec ses principes de naissance et de mort, d'amour et de maternité $»^{37}$. Ce choix la rend coupable aux yeux de Tolstoj et c'est pourquoi elle doit mourir. Ainsi que l'a affirmé Lev Chestov, la mort cruelle réservée à Anna par Tolstoj est en fait le prix que l'héroïne 
tragique dut payer pour l'écrivain lui-même - aux prises dès le départ avec le principal sujet de son roman - l'accès à une paix intérieure :

Si Anna avait pu (...) mourir non pas écrasée [razdavlennoj] et anéantie, mais fière et droite, Tolstoj aurait perdu le pivot qui lui permettait de maintenir son équilibre spirituel. Il était face à une alternative - Anna ou lui-même, sa destruction à elle ou son salut à lui. Et il sacrifia Anna, qui s'était tournée vers Vronskij alors que son mari était en vie. ${ }^{38}$

Du point de vue de l'orthodoxie - à la fois si proche et si distante pour Tolstoj - la tentation érotique est effectivement un obstacle au chemin de l'homme vers Dieu et la perfection spirituelle. Cette perfection inclut un refus de ce qu'offre le Monde - ce Monde étant déchu et pécheur - au nom d'une vie future et meilleure. Bien que les chemins de Tolstoj et de l'Église russe se soient séparés, il ne pouvait se soustraire à l'influence de telles conceptions sur sa pensée. Le cœur de la pensée de Tolstoj a toujours été orthodoxe, quelques différents qu'aient pu être ses enseignements de ceux de l'Église.

\section{De l'hostilité à l'obsession}

Irving Singer soutient avec raison que «nulle part dans ses écrits Tolstoj ne représente le sexe - même sous son meilleur jour - comme une union favorable ou une cause d'harmonie " ${ }^{39}$. Déjà dans Le Bonheur conjugal et dans Guerre et Paix, Tolstoj vante les femmes se dévouant entièrement à leur famille (Natacha, l'un des personnages favoris de Tolstoj dans ce dernier roman, est un exemple de mère et d'épouse vertueuse car elle « elle n'a plus ni idées ni expressions à elle, et c'est toujours Pierre qui parle par sa bouche $»^{40}$ et car les " propos et ratiocinations concernant les droits des femmes non seulement ne l'intéressent pas, mais elle ne les comprend absolument pas ${ }^{41}$ ), tout en se montrant explicitement sévère envers le personnage "hypersexualisé » de la Comtesse Hélène Bezukhov (Hélène Kuragin). Dans l'univers de Tolstoj, explique Hugh Mc Lean, «Hélène est l'incarnation de la dépravation féminine. Elle a eu une relation incestueuse avec son frère, elle épouse Pierre pour son argent sans le moindre sens de l'engagement; pour elle, l'adultère est un mode de vie naturel ; et, peut-être pire que tout, elle fait quelque chose, on ne sait trop quoi (un avortement?) pour s'empêcher de devenir mère $»^{42}$.

Non sans raison, Tolstoj fait disparaître la Comtesse Bezukhov de son roman quelque peu brusquement: il lui attribue une mort dépourvue de sens, la maladie dont elle meurt n'étant jamais clairement déterminée, et ne la mentionne ensuite plus, comme si elle n'avait jamais existé.

À dater de la publication du Bonheur conjugal et du monumental Guerre et Paix, l'attitude de l'écrivain à l'égard des femmes qui ont un caractère indépendant et un appétit sexuel assumé devint de plus en plus hostile. Considérant le cas d'Anna Karénine ou de l'également insatisfaite Macha du Bonheur conjugal, on est amené à voir Tolstoj comme approuvant certains des espoirs déçus, des aspirations ou des besoins physiques des femmes. D'un autre côté, comme le soutient Cook, «il n'y a guère de doute en ce qui concerne la personne à laquelle il estime que revient le pouvoir. Pour lui, la clef d'un mariage réussi est déjà la maîtrise par le mari de l'esprit d'indépendance de sa femme un processus dans lequel l'accouchement joue un rôle important $»^{43}$. 

de rester libre, mais Tolstoj n'hésite pas à présenter le meurtre comme un moyen extrême pour les hommes de maintenir leur pouvoir sur la sexualité des femmes. La scène du meurtre est présentée dans le roman avec une satisfaction sexuelle évidente.

列 faut faire, Ma Religion et Sonate à Kreutzer, il est possible de suivre le développement de la misogynie de Tolstoj et de sa consolidation finale en une haine des femmes "peut-être à la suite de la déception liée à son propre mariage » et à un sentiment de culpabilité «de ses propres instincts sexuels puissants $»^{44}$. Tolstoj a toujours ressenti de la honte et du malaise à l'égard de ses désirs sexuels. Il semble néanmoins qu'il ait choisi une manière assez curieuse de vivre cette honte : il blâmait et parfois même calomniait les femmes, les rendant responsables de sa propre incapacité à résister à ses élans érotiques. Les femmes souhaitant mener une vie sexuelle libre en sacrifiant la maternité devinrent sa cible de prédilection. En tant qu'homme, Tolstoj n'a jamais réussi à gérer l'effet que les femmes exerçaient sur lui, et son écriture était donc devenue un moyen de compenser ses névroses et obsessions à l'égard du sexe opposé. Il n'est guère surprenant que «la plus grande résistance aux penchants sexuels ait été constatée chez quelqu'un aux élans charnels si puissants et omniprésents ${ }^{45}$.

Il convient de souligner que, travaillant sur Anna Karénine, Tolstoj consacra un temps exceptionnel à comprendre et à prendre en pitié une "femme déchue ». Nous savons qu'au cours du fastidieux travail de relecture (le roman fut presque constamment modifié et corrigé durant sa production), Tolstoj a progressivement transformé Anna de la courtisane antipathique qu'elle était initialement en l'héroïne tragique que nous connaissons aujourd'hui : "aimant Anna, il combattait son penchant rationnel le pressant de la condamner pour avoir enfreint les règles $»^{46}$. On peut soutenir qu'il s'agit là de l'une des dernières marques d'indulgence de la part de Tolstoj. Dans Ce qu'il faut faire, l'écrivain s'adressait aux "femmes déchues » en usant d'un vocabulaire vulgaire qui est encore censuré dans certaines éditions de cette œuvre :

Il est des femmes humaines présentant les qualités les plus nobles de l'être humain et il en est d'autres qui sont des putains. (...) Toute femme, quelle que soit la manière dont elle s'habille et quelque raffinée qu'elle puisse être, qui refuse la maternité sans s'abstenir de relations sexuelles est une putain. Et quelque déchue que puisse être une femme, si elle se dévoue consciemment à donner la vie, elle assume le meilleur et le plus noble service qui soit - elle accomplit la volonté du Seigneur - et nul ne lui est supérieur. ${ }^{47}$.

Dans Sonate à Kreutzer, Tolstoj pose un idéal encore plus redoutable : on devrait sans doute renoncer à toute forme de relation avec les femmes, parce que ce sont les femmes qui tirent délibérément avantages de leurs corps tentateurs pour réduire les hommes en esclavage. Les hommes s'imaginent naïvement qu'ils contrôlent les femmes, alors que c'est plutôt l'inverse qui se produit: "ces odieux jerseys, ces tournures et qu'on étale ses épaules, ses bras et ses seins nus » [SK, 55] - se plaint Tolstoj. Adoptant une opinion clairement décadento-misogyne, Tolstoj en vint à considérer l'apparence attirante des femmes comme une stratégie délibérée des femmes pour asseoir leur pouvoir sur les hommes : «Les femmes, comme les Juifs, se paient de la servitude en laquelle elles ont été réduites en asservissant les hommes. Et nous l'avons bien mérité. Il ne faut pourtant pas céder, mais bien éliminer en soi la faiblesse par laquelle elles nous tiennent. $\gg^{48}$ À l'époque où il écrit Sonate à Kreutzer, Tolstoj est déjà complètement obsédé par la sexualité des femmes qui est, dans son 
esprit, devenue un défi physique, moral et philosophique gigantesque. Les femmes se sont mises à représenter l'hypocrisie, la tentation pécheresse et la débauche ostentatoire, indépendamment de leur statut social: "D'après vous, les femmes de notre société ont d'autres intérêts que les femmes tombées? Je prétends que non. En voici la preuve. (...) Mêmes toilettes, mêmes manières, mêmes parfums, même étalage de bras, d'épaules et de seins (...). » [SK, 56] Edward Greenwood peut donc affirmer avec raison que "le propos central de Sonate à Kreutzer n'est pas tant une attaque contre l'aspect physique du sexe (...) que la volonté ardente de se libérer de tout lien avec une femme $»^{49}$. Fasciné par la figure du Christ - pauvre enseignant errant et célibataire Tolstoj a fini par ne plus approuver le mariage (et la famille) qu'à peine (Sonate à Kreutzer rejette le mariage comme un vulgaire alibi à la même corruption qui pervertit les relations sexuelles non conjugales), et, s'il le fait, c'est en envisageant un mariage absolument chaste. Il est intéressant de noter qu'aussi bien dans Guerre et Paix que dans Anna Karénine, Tolstoj soumet ses couples mariés favoris (Pierre et Natacha d'une part, Levin et Kitty d'autre part) à un sentiment de "honte " ressenti durant leur lune de miel. Alors qu'il écrit Anna Karénine, la vie privée du « sage de Jasnaja Poljana » devient déjà une tragi-comédie faite de comportements de plus en plus étranges (une abstention totale de relations sexuelles avec sa femme, par exemple) et de terribles disputes conjugales trouvant leur origine dans l'infidélité chronique de Tolstoj (!), qui s'accompagne d'un sentiment général de culpabilité qu'il décrivait continuellement dans son Journal et dont il se servait pour harceler (sa femme) - Sophie.

\section{Conclusion}

L'objectif de cette étude était de traiter les convictions de Tolstoj au sujet de l'amour charnel. La lecture d'Anna Karénine et d'autres œuvres de Tolstoj permet de voir en l'écrivain russe le prédicateur d'un philosophie de l'amour extrêmement rigide et conservatrice, dans laquelle le sexe est exclusivement considéré soit comme un moyen de reproduction, soit comme quelque chose qui doit être totalement réprimé dans l'intérêt d'un mariage sain unissant les partenaires par un lien spirituel quasi-fraternel.

Tous les éléments clefs de la philosophie tolstojenne de l'amour charnel se retrouvent dans Anna Karénine. Pour illustrer sa thèse voulant que la passion charnel ne peut mener qu'à la perdition, Tolstoj fait usage dans ce roman d'une variété de figures de style et appuie ses opinions sur un raisonnement philosophique partagé avec le fondateur du christianisme - Jésus de Nazareth, avec des penseurs russes tels que Dostoevskij et Solov'ëv, et des philosophes européens tels que Schopenhauer, Rousseau et Strindberg. À mon avis, il avait aussi perçu le caractère effrayant de l'érotisme, son lien avec la douleur et la mort, avec la pulsion de blesser et d'être blessé, telle qu'elle avait déjà été observée par le Marquis de Sade et les écrivains décadents, et qu'elle se refléterait plus tard, au XX ${ }^{e}$ siècle, par Bataille.

À partir de ces influences variées et en se fondant sur son propre vécu, Tolstoj a élaboré sa curieuse thèse voulant que le sexe fasse obstacle au bonheur humain et à l'évolution naturelle de la vie. Le sexe est en lien avec la mort, sauf s'il sert la cause de la reproduction ou de l'élévation spirituelle des partenaires. Et que pourrait être cette « élévation spirituelle » ou « communion »? Il s'agirait probablement de la situation où le sexe n'est plus désirable: "Le bonheur commence après que [les] couples ont abandonné l'espoir de trouver le plaisir dans l'activité sexuelle entre eux. ${ }^{50}$ 
Aux yeux du lecteur moderne, les idées de Tolstoj quant à l'amour charnel paraissent entièrement dépassées, et portent peut-être même atteinte à la crédibilité d'un écrivain au talent par ailleurs indiscutable. Pourtant, si Tolstoj est aujourd'hui tourné en ridicule pour avoir prêché son éthique d'ascétisme céleste incompatible avec les besoin physiques de l'être humain, s'il nous est si facile de croire qu'il n'a condamné Anna Karénine à la destruction que sur la base de sa vision faussée de la vie sexuelle, d'où vient cette idée déjà ancienne, si souvent exprimée dans l'histoire de la littérature et si passionnément développée par Bataille, que le monde érotique est un monde de douleur, de détresse, de déchéance et de mort? Ainsi que l'écrit ce dernier: «l'érotisme est un fardeau trop lourd pour l'homme ». Peut-être Tolstoj avait-il ainsi perçu la problématique de l'érotisme et était arrivé à une conclusion semblable. Pourtant, contrairement à Bataille, et conformément à ses idées religieuses, il avait trouvé dans l'ascèse extrême la seule échappatoire aux voies dangereuses de l'érotisme.

\section{NOTES}

1. Compte tenu de la notoriété des œuvres de Lev N. Tolstoj, les titres de celles-ci (et leurs protagonistes) seront donnés en français dans l'ensemble de l'article.

2. Mc Lean H., In Quest of Tolstoy (En quête deTolstoj), Academic Studies Press, Brighton, 2008, p. 106.

3. Voir : Praz M., La Chair, la Mort et le Diable : le romantisme noir, Gallimard/Tel, Paris, 1998 ; Janion M., "Cień "boskiego markiza" »(L’ombre du « Divin marquis »), in : GorĄczka romantyczna (La Fièvre romantique), Universitas, Cracovie, 2000 ; Hutnikiewicz A., Twórczość literacka Stefana Grabińskiego 1887-1936 (L'ÆEuvre littéraire de Stefan Grabiński), Towarzystwo Naukowe w Toruniu, Toruń, 1959 ; Kruszelnicki M., Oblicza strachu. Tradycja i współczesność horroru literackiego (Les visages de la peur. La tradition et l'actualité de l'horreur littéraire), Wyd. Adam Marszałek, Toruń, 2003, 2010.

4. Écrit en français en 1887-1888, publié pour la première fois en allemand en 1893.

5. Sur se sujet voir: Eikhenbaum B., Lev Tolstoj. Semidesjatye gody (Lev Tolstoj. Les années soixante-dix), Izdatel'stvo Sovetskij Pisatel', Leningrad, 1960, pp. 125 \& 152; Meyer P., "Anna Karenina : Tolstoy's Polemic with 'Madame Bovary' (Anna Karénine : la polémique de Tolstoj avec Madame Bovary), in : Russian Review, t. LIV, cahier 2, 1995, pp. 243-259.

6. Tolstoy's Diaries (22 December 1893) (Le Journal de Tolstoj [22 décembre 1893]), t. I : 1847-1894, édité et traduit par Christian F. R., The Athlone Press, Londres, 1985, p. 324. Sauf mention contraire, toutes les traductions sont de l'auteur de l'article et du traducteur de celui-ci.

7. Troyat H., Tolstoï, Fayard, Paris, 1965, 893 p. ; Bulgakov V., L. N. Tolstoj v poslednij god ego žyzni (Tolstojdans la dernière annéede sa vie), Goslitizdat, Moscou, 1957 ; Johnson P., Intellectuals. From Marx and Tolstoy to Sartre and Chomsky (Intellectuels. De Marx et Tolstoj à Sartre et Chomsky), Harper and Row Publishers, New York, 1988.

8. Cook T., "Introduction », in : Tolstoy L., The Death of Ivan Ilych and Other Stories (La mort de Ivan Ilyč et autres histoires), traduit par A. Maude et J. D. Duff, Wordsworth Editions Limited, New York, 2004, p. XIV.

9. Tolstoï L., Ma Confession, traduction de Zoria, Albert Savine, Paris, 1887. 
10. Stepun F., "The Religious Tragedy of Tolstoy ", in : Russian review, t. XIX, cahier 2, 1960, p. 169.

11. Pour plus d'informations sur le conflit entre Tolstoj et l'Église orthodoxe, voir : Kolstø P., "The Demonized Double: The Image of Lev Tolstoy in Russian Orthodox Polemics " (L'alter ego démonisé : l'image de Lev Tolstoj dans les polémiques avec l'Orthodoxie russe), in : Slavic Review, t. LXV, cahier 2, 2006, pp. 304-324.

12. Jahn G. R., "The Image of the Railroad in Anna Karenina " (L'image du chemin de fer dans Anna Karénine), in : The Slavic and East European Journal, t. XXV, cahier 1, 1981, pp. 2-8.

13. Tolstoï L., Anna Karénine, t. I (traducteur non mentionné), Hachette, Paris, 1896, p. 86. Les prochaines occurences seront notées dans le texte par [AK] suivi du numéro de tome et de la page.

14. Tussing Orwin D., Tolstoy's Art and Thought 1847-1880 (L'artet la pensée de Tolstoj 1847-1880), Princeton University Press, Princeton, 1993, p. 184.

15. Šestov L., Dobro v učenii grafa Tolstogo i F. Nitše (L'Idée du bien chez Tolstoj et Nietzsche), M. V. Pirozhkova, Saint-Pétersbourg, 1907, p. 4.

16. Ibid., pp. 215-216.

17. Whitcomb C., "Treacherous 'Charm' in Anna Karenina " (Le « charme » trompeur dans Anna Karénine),in : The Slavic and East European Journal, t. xxxIX, cahier 2, 1995, p. 217.

18. Clifton Edwards G., « Tolstoy », in : The Sewanee Review, t. IX. Cahier 4, 1901p. 465.

19. Cruise E., «Women, Sexuality, and Family in Tolstoy (Les femmes, la sexualité et la famille chez Tolstoj), in: The Cambridge Companion to Tolstoy, sous la direction de Tussing Orwin D., Cambridge University Press, Cambridge, 2002, p. 201.

20. Tolstoï L., Ce qu'il faut faire, traduit par B. Tseytline et E. Jaubert, Albert Savine, Paris, 1888, p. 275.

21. Christian F. R., Tolstoy. A Critical Introduction (Tolstoj. Une introduction critique), Cambridge University Press, London, 1969, p. 176.

22. Ibid., p. 173.

23. Tolstoï L., Sonate à Kreutzer, traduit par E. Halpérine-Kaminsky, Flammarion, Paris, 1906, pp. 93-94. Les prochaines occurences seront notées dans le texte par [SK] suivi du numéro de page.

24. Pour plus d'information sur les liaisons entre Anna Karenina et Sonate à Kreutzer voir: Aldanow M., Zagadka Tołstogo (L'énigme Tolstoj), Brown University Slavic Reprint Series, Providence, 1969, pp. 39-56.

25. Tussing Orwin D., op. cit., p. 186.

26. Ibid., p. 319.

27. Nous trouvons cette expression dans l'original russe et dans la traduction anglaise, mais l'expression n'est pas reprise pas dans la traduction française.

28. Soloviev Vl., The Meaning of Love (Le sens de l'amour), traduit par T. R. Beyer, Lindisfarne Book, New York, 1995, p. 21.

29. Ibid., p. 22.

30. Tolstoï L., Ma Religion (traducteur non mentionné), Fischbacher, Paris, 1885, p. 82.

31. Bataille G., « Le coupable », in : Euvres complètes, t. v : La Somme Athéologique, Gallimard, NRF, Paris, 1973, p. 247

32. Ibid., p. 267

33. Voir : Bataille G., L'Érotisme, Les Éditions de minuit, Paris, 1957, 312 p. et Les larmes d'Éros, 10| 18, Paris, 2004, $125 \mathrm{p}$.

34. Wasiolek E., Tolstoy's Major Fiction (La Grande fiction de Tolstoj), University of Chicago Press, Chicago, 1978,p. 140.

35. Markovitch M. I., Jean-Jacques Rousseau et Tolstoï, Champion, Paris, 1928.

36. Lönnquist B., « Anna Karenina », in : The Cambridge Companion to Tolstoy, op. cit., pp. 89 \& 93. 
37. Augustus Maninng Cl., «Tolstoy and Anna Karenina » (Tolstoj et Anna Karénine), in : PMLA, t. XLII, cahier 2, 1927, p. 515.

38. Šestov L., op. cit., p. 4.

39. Singer I., The Nature of Love: The Modern World (Lanature de l'amour : lemonde moderne), MIT Press, Cambridge, 2009, p. 59.

40. Tolstoï L., La guerre et la paix, t. III, traduit par une russe [sic], Hachette, Paris, 1884, p. 408

41. Nous trouvons ce passage dans l'original russe de Guerre et Paix (Epilogue, 1 ere partie, Chapitre 10) et dans la traduction anglaise, mais il n'est pas repris pas dans la traduction française.

42. Mc Lean H., op. cit., p. 116.

43. Cook T., op. cit., p. XIII.

44. Ibid., p. XVI.

45. Singer I., op. cit., p. 60.

46. Christian F. R., op. cit., p. 179.

47. Tolstoy L., What Then Must We Do?(Ce qu'il faut faire), traduit par A. Maude, Oxford University Press, Londres, 1942, p. 170.

48. Tolstoy's Diaries (19 July 1893) (Journal de Tolstoj [19 juillet 1893]), op. cit., p. 328.

49. Baker Greenwood E., Tolstoy. The Comprehensive Vision (Tolstoj. La vision globale), Methuen \& Co. Ltd, Londres, 1980, p. 140.

50. Singer I., op. cit., p. 59.

\section{RÉSUMÉS}

Le présent article vise à reconstruire la vision qu'avait Lev N. Tolstoj de l'amour physique et de faire de celle-ci une évaluation critique. Aux yeux du lecteur moderne, ses opinions peuvent apparaître totalement dépassées, scandaleuses, ou peut-être même porter atteinte au crédit d'un écrivain dont les qualités artistiques sont par ailleurs incontestables. Face aux points de vue défendus par Tolstoj, il est parfois nécessaire de faire preuve de beaucoup de compréhension et de mansuétude, surtout de la part des lectrices! De mon point de vue, ils restent cependant dignes d'intérêt sur le plan philosophique. Anna Karenina (Anna Karénine) est l'objet principal de mon attention parce que c'est dans cette œuvre que Tolstoj consacre à la relation sexuelle une attention extraordinaire, philosophique. Je m'attarderai cependant également sur des romans tels que Semejnoe sčastie (Le Bonheur conjugal), Vojna i Mir (Guerre et Paix) et Krejtcerova sonata (Sonate à Kreutzer), ainsi que sur les essais philosophique Tak čto že nam delă̌? (Ce qu'il faut faire) et Moja Vera (Ma Religion), afin de pouvoir montrer comment l'hostilité de Tolstoj envers les passions charnelles et, plus généralement, envers les femmes a évolué pendant les vingt années séparant la publication de Vojna i Mir (1869) de celle de Krejtcerova sonata (1889). L'article couvre également les causes sociales, philosophiques et personnelles de l'aversion grandissante de Tolstoj pour l'amour physique. 
INDEX

Index géographique : Russie

oeuvretraite Tolstoj. L. N. : Anna Karenina, Tolstoj. L. N. : Krejtcerova sonata, Tolstoj. L. N. : Moja Vera, Tolstoj. L. N. : Semejnoe sčastie, Tolstoj. L. N. : Tak čto že nam delǎ̌?, Tolstoj. L. N. : Vojna i Mir

Mots-clés : érotisme, femmes, littérature russe, mort, sexe

Index chronologique : XIXe siècle

\section{AUTEURS}

\section{MICHAt KRUSZELNICKI}

Institut de Philosophie, Université de Basse-Silésie, Wrocławmikrusz@gmail.com 